\title{
PG3, A field in the Bulge of Our Galaxy: Description of a Galactic Model
}

\author{
Y.K. Ng ${ }^{1, *}$, G. Bertelli ${ }^{2,3}$, C. Chiosi $^{3}$ \\ A. Bressan ${ }^{4}$, H. Habing ${ }^{1}$ and R.S. Le Poole ${ }^{1}$ \\ ${ }^{1}$ Leiden Observatory, Leiden, the Netherlands \\ ${ }^{2}$ National Council of Research, Rome, Italy \\ ${ }^{3}$ Department of Astronomy, Padua, Italy \\ ${ }^{4}$ Astronomical Observatory, Padua, Italy
}

\begin{abstract}
Preliminary results are shown of our study on the metallicities and ages of the stellar populations present in different components along the line of sight to field \#3 of the Palomar-Groningen Variable Star Survey (PG3) with synthetic Hertzsprung-Russell Diagrams (HRDs).
\end{abstract}

\section{Model Building}

Wesselink (1987) re-examined the variable stars in PG3 (Larsson-Leander $1959 ; l=0^{\circ}, b=-10^{\circ}$ ) by measuring $\mathrm{B}_{J}$ and $\mathrm{R}$ Schmidt plates on the Leiden Astroscan (de Vries 1986). Wesselink's work is extended to all stars in PG3 ( $\mathrm{Ng}$ et al. 1990, 1992). A CMD has been obtained for a great many stars stars in an area sized $3.5 \times 3.5$ centered around $l=1^{\circ}$ and $b=-11^{\circ}$. A first analysis ( $\mathrm{Ng}$ et al. 1992) with synthetic CMDs (Chiosi et al. 1988) showed that the contamination with the foreground stars is significant. With a more elaborated version of the CMD synthesis tool with a continuous metallicity coverage the analysis is continued. Four datasets from the latest models computed (Bertelli et al. 1990; Bressan et al. 1192; Alongi et al. 1992) with $Z=\{0.0004,0.001,0.008,0.02\}$ are the basis for the continuous $\mathrm{Z}$ synthetic HRD generator. Inputs to start the synthesis engine are

1) the slope for the powerlaw IMF (we used the Salpeter value);

2) the time interval for and the shape of the star formation rate assumed;

3 ) the range in $\mathrm{Z}$ (for simplicity a linear increase in time is assumed).

The B, R magnitudes for the synthetic stars are transformed into the photographic $B_{J}, R$ magnitudes (Blair and Gilmore 1983). Synthetic HRDs are generated and 'observed' through an HRD Software Telescope which contains a two component galactic model: a spheroidal component (age: 17-15 Gyr, $\mathrm{Z}=0.0004-0.0156$ ) and a disk component (age: 15-8 Gyr, $\mathrm{Z}=0.009$ 0.02 , scalelength $3.5 \mathrm{kpc}$ and scaleheight $200 \mathrm{pc}$ ). Realistic features of the HRD Software Telescope are the reddening of the synthetic star, the inclusion of apparent (unresolved) photometric binaries and the simulation of photometric errors as a function of magnitude for each passband.

*

The research of Y.K. Ng is supported under grant 782-373-040 by the Netherlands Foundation for Research in Astronomy (ASTRON) which receives its funds from the Netherlands Organization for Scientific Research (NWO). 


\section{Preliminary Results and Discussion}

Synthetic stars, with a limiting magnitude $\mathrm{B}_{J, l i m}=19^{\mathrm{m}}$, are generated for a two component (spheroid, disk) Galaxy with a ratio Spheroid : Disk = $3: 4$. We adopted $R_{0}=8 \mathrm{kpc}$ for the distance to the Galactic Centre and a mean reddening $\mathrm{E}\left(\mathrm{B}_{J}-\mathrm{R}\right)=0{ }^{m} 15 \pm 0{ }^{m} 05$ (Wesselink, 1987). The reddening for a synthetic star with $|z|<90 \mathrm{pc}$ is assumed proportional to its distance from the Sun. In the figures 1 and 2 we show the results of star counts in different magnitude and colour bins. The effects due to photometric errors and unresolved apparent binaries are not considered.

Figure 1 shows the synthetic color distribution for the individual, combined and and the observed color distribution (solid line $=$ combined, dashed line $=$ spheroid, dot dashed line $=$ disk, circles $=$ observed .

Figure 2 shows the synthetic starcounts for the combined and individual components together with the observed starcounts (solid line $=$ combined, dashed line $=$ spheroid, dot dashed line $=$ disk, circles=observed).

The color distribution of the stars in PG3, figure 1, is primarily governed by the scaleheight of the disk main sequence stars. The evolved stars require an additional contribution by the spheroidal component. The adopted metallicity and age dispersion for the spheroidal and disk component determines the width of the color distribution. Figure 2 shows that the color equations used (Blair and Gilmore 1982) aren't optimal because in each figure the shape of the synthetic starcounts resembles more closely the observed data in the next figure. Bessel (1986) pointed out that cubic transformations should be used, because the extended red tail of the Kron-Cousins $\mathrm{R}$ band differs from the photographic $\mathrm{R}$ band. In figure 2 the peak near $\mathrm{B}_{J}=16^{m} \cdot 5$ in the observational data in the slice for $\mathrm{B}_{J}-\mathrm{R}=[1.2 ; 1.4]$ is a signature of the horizontal branch from the spheroidal component and it holds information about the metallicity and the history of star formation. The simulated starcounts are one magnitude fainter than the observed data. Uncertainties in the theoretical models and the assumed metallicity range can result in a 0.5 magnitude shift while the shift from the color transformation is less than 0.5 magnitude. The reddening tends to shift the simulated starcounts to fainter magnitudes because the adopted value is regarded as a lower limit. This result suggests that the distance to the Galactic Centre is smaller than the $8 \mathrm{kpc}$ adopted. Some future improvements on the galactic model description and a check on the theoretical evolution models are required before any definitive conclusions can be made.

\section{References}

Alongi, M. et al., 1992, Astron. Astrophys Suppl. Ser., submitted Bertelli, G. et al., 1990, Astron. Astrophys. Suppl. Ser. 85, 845-853

Bessell, M.S., 1986, P.A.S.P. 98, 1303-1311

Blair, M. and Gilmore, G., 1982, P.A.S.P. 94, 742

Bressan, A. et al., 1992 , in preparation

Chiosi, C. et al., 1988, Astron. Astrophys. 196, 84

Larsson-Leander, G., 1959, Proc. IAU symposium 7, 22

$\mathrm{Ng}$, Y.K. et al., 1990, Proc. ESO/CTIO Workshop on 'Bulges of Galaxies', B.J. Jarvis and D.M. Terndrup (eds.), 89-92

$\mathrm{Ng}, \mathrm{Y}, \mathrm{K}$. et al., 1992, Proc. IAU symposium 149, 'Stellar Populations of Galaxies',

B. Barbuy and A. Renzini (eds.), 462

Vries, de C., 1986, Ph.D. thesis, Leiden University, the Netherlands

Wesselink, T.J.H., 1987, Ph.D. thesis, Nijmegen University, the Netherlands 

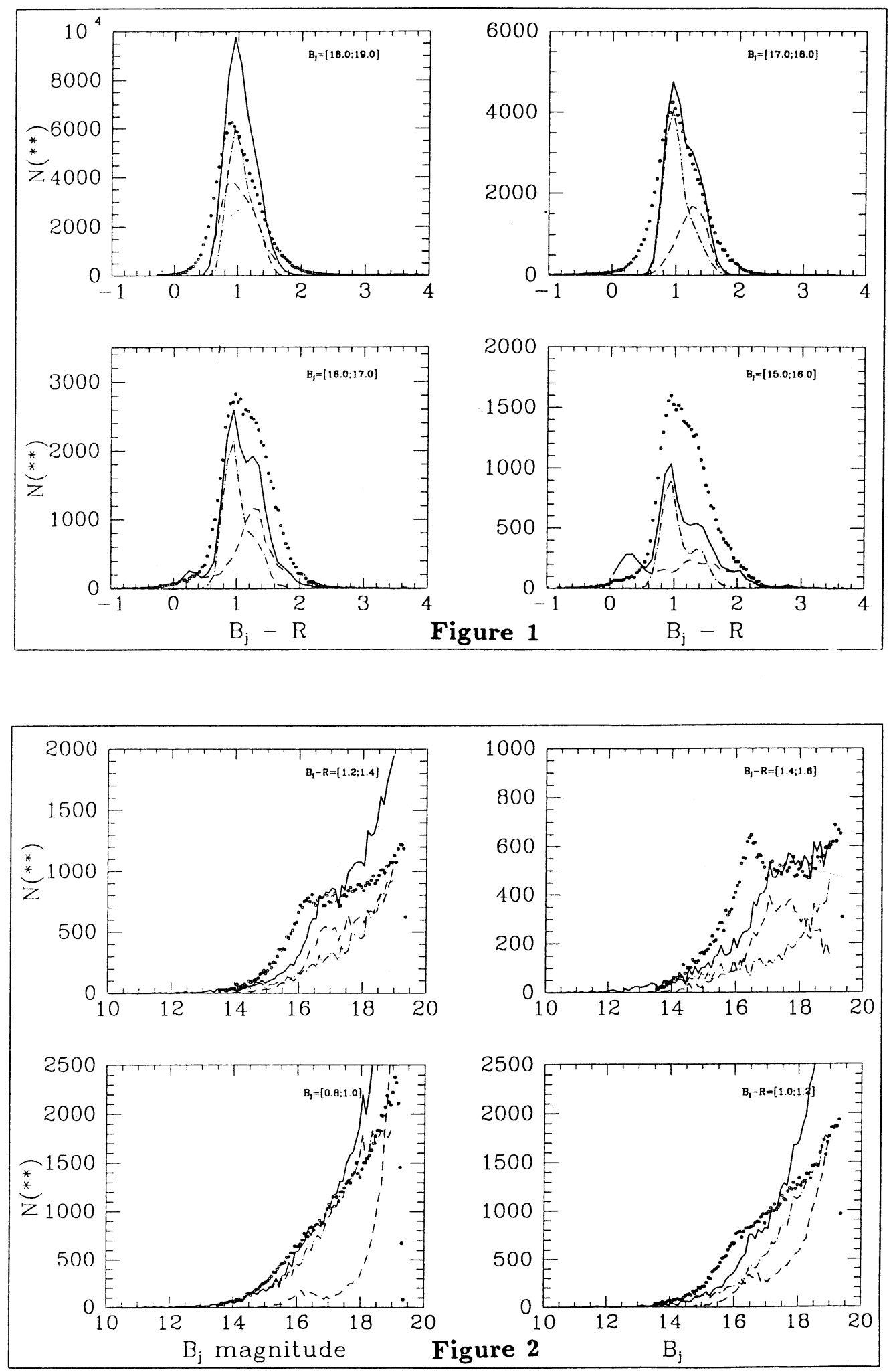


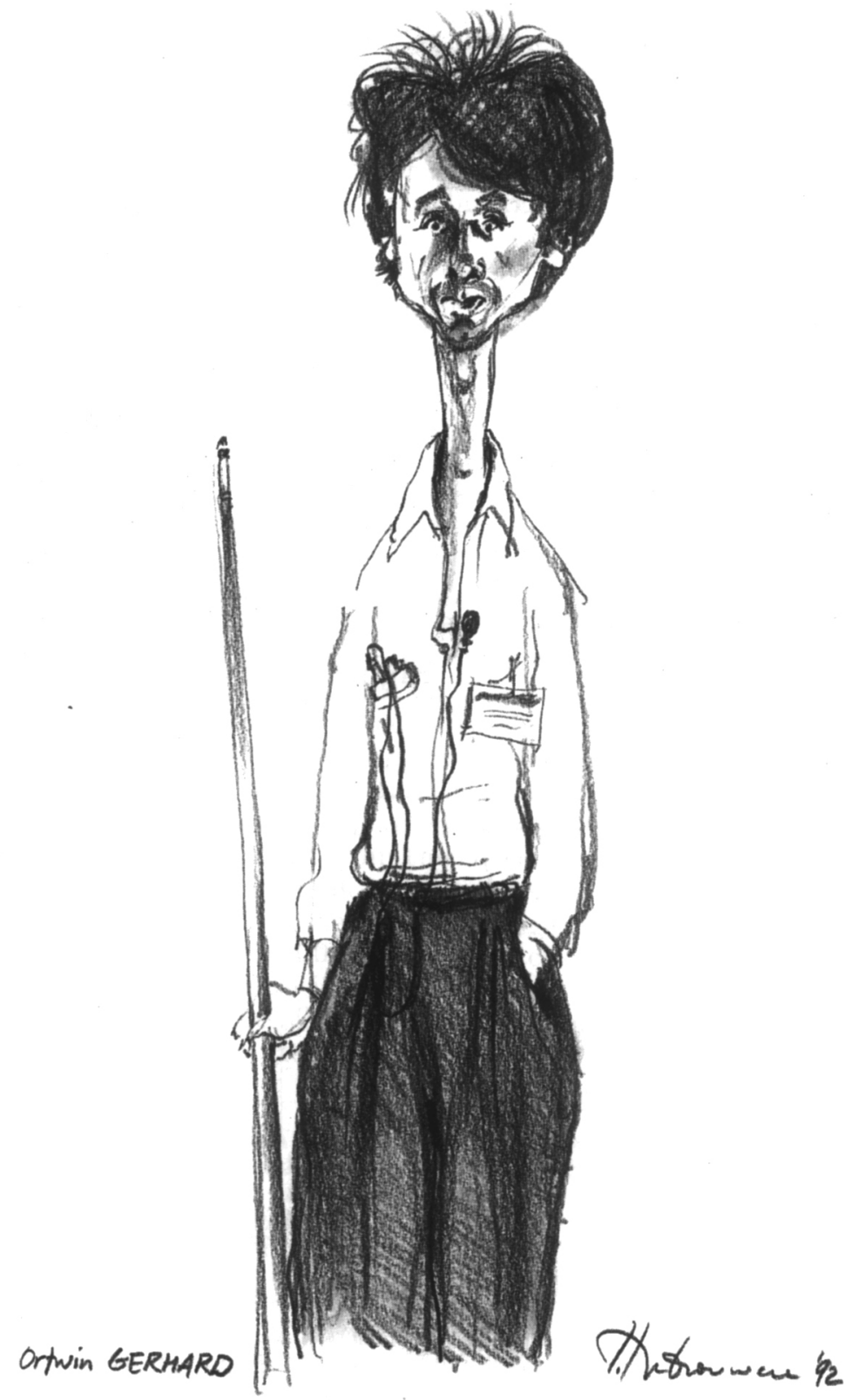

